\title{
DATA-DRIVEN COMBUSTION MODELING FOR A TURBULENT FLAME SIMULATED WITH A COMPUTATIONALLY EFFICIENT SOLVER
}

\author{
Mohsen Talei, Man-Ching Ma, Richard Sandberg \\ Department of Mechanical Engineering \\ The University of Melbourne \\ Parkville, Victoria, 3010, Australia \\ Email: mohsen.talei@unimelb.edu.au
}

\begin{abstract}
The use of machine learning (ML) for modeling is on the rise. In the age of big data, this technique has shown great potential to describe complex physical phenomena in the form of models. More recently, ML has frequently been used for turbulence modeling while the use of this technique for combustion modeling is still emerging. Gene expression programming (GEP) is one class of ML that can be used as a tool for symbolic regression and thus improve existing algebraic models using high-fidelity data. Direct numerical simulation (DNS) is a powerful candidate for producing the required data for training GEP models and validation. This paper therefore presents a highly efficient DNS solver known as HiPSTAR, originally developed for simulating non-reacting flows in particular in the context of turbomachinery. This solver has been extended to simulate reacting flows. DNSs of two turbulent premixed jet flames with different Karlovitz numbers are performed to produce the required data for training. GEP is then used to develop algebraic flame surface density models in the context of large-eddy simulation (LES). The result of this work introduces new models which show excellent performance in prediction of the flame surface density for premixed flames featuring different Karlovitz numbers.
\end{abstract}

\section{NOMENCLATURE}

c Progress variable

$D$ Pipe diameter

fit Fitness of the individual $k$ Fourier mode

$l_{r} \quad$ Radial distance

$l_{z} \quad$ Streamwise distance

$\mathrm{Ka}$ Inlet Karlovitz number

$L_{f} \quad$ Average flame length

$M$ Inlet Mach number

$N_{r} \quad$ Number of points in the radial direction

$N_{z} \quad$ Number of points in the streamwise direction

$r$ Radial position

$R$ Pipe radius

$R e_{D}$ Reynolds number

$S_{L} \quad$ Laminar flame speed

$S_{c} \quad$ Consumption speed

$T$ Gas temperature

$T_{u} \quad$ Unburnt gas temperature

$T_{b} \quad$ Burnt gas temperature

$U_{b} \quad$ Inlet bulk velocity

$u^{\prime}$ Velocity fluctuations

$z \quad$ Streamwise position

$\alpha \quad$ Heat release parameter, $\left(1-T_{u} / T_{b}\right)$

$\delta_{z} \quad$ Zel'dovich flame thickness

$\delta_{L} \quad$ Thermal flame thickness

$\Delta$ Filter size

$\phi$ Equivalence ratio

$\theta \quad$ Reduced temperature

$\Sigma$ Velocity fluctuations

$\dot{\omega}_{\theta} \quad$ Filter size

$\rho_{0} \quad$ Unburnt gas density 
$\Sigma_{\text {gen }} \quad$ Generalised flame surface density

$\Sigma_{\text {gen }}^{D N S} \quad$ Generalised flame surface density obtained from DNS

$\Xi_{\text {gen }}$ Wrinkling factor

... LES filtered variable

$\langle\ldots\rangle_{s} \quad$ Averaged quantity over the flame surface

$\approx$ Favre-average quantity

$\langle\ldots\rangle$ Volume averaged quantity

$\langle\ldots \mid \ldots\rangle$ Conditional averaged quantity

\section{Introduction}

"Learn from experience" [1] can be used as a definition of machine learning (ML) in simple terms. In the past 70 years, this technique has been regularly used to find patterns in the data and make decisions or undertake predictions based on the found patterns. Thanks to the advances in computational power and the increased amount of available data, ML has provided tremendous opportunities for describing physical processes in the form of models. More recently, ML has found its way into the field of fluid mechanics, in areas such as flow modeling and flow optimization and control [2].

ML methods can be divided into three groups. In the first group, referred to as unsupervised learning, ML is used to find patterns in the data without providing any models that relate input parameters to output variables. Methods such as proper orthogonal decomposition (POD) and principal component analysis (PCA) are in this category. On the other side of the spectrum, supervised learning produces a model for predicting certain output variables as a function of the inputs to the model. Neural networks and evolutionary algorithms belong to this group of ML. In the third group, called semi-supervised learning, the model is trained using a limited amount of data. Therefore, the algorithm has an important role to play in producing a reasonably accurate model. Methods such as generative adversarial networks and reinforcement learning fall in this category.

In the field of flow modeling, neural networks have received significant attention, e.g. [3-5]. This is due to their ability to model complex problems, thereby enhancing our predictive capabilities. Gene expression programming (GEP), classified under evolutionary algorithms, has also been used for developing turbulence models [6]. In this context, GEP is used as a symbolic regression tool to produce a functional form for the model. The advantage of such technique over neural networks approaches is that the produced algebraic model clearly shows the relationship between inputs and outputs, it can be easily implemented into a solver and constraints can be imposed to ensure that the model is in agreement with the fundamental physics of the problem. In most cases, high-fidelity simulation data such as direct numerical simulation (DNS) are used to train models for the unresolved terms in the lower-fidelity simulations. GEP has already shown promising results in Reynolds-averaged Navier-Stokes (RANS) and large-eddy simulation (LES) modeling, e.g. [6-9].
ML has been recently used in a few recent studies for combustion modeling [10-18]. Several groups have used ML for dimensional reduction and produced improved predictions of the presumed probability density functions (PDFs) or flamelet manifolds $[11,16,17]$. Another group of studies has used neural networks to model unresolved terms in the LES formalism [10,12]. For instance, the study by [12] used the convolutional neural network (CNN) method to model the unresolved terms in the filtered transport equation of the progress variable while the study by Lapeyre et al. [10] used CNN to model the flame surface density (FSD), both showing encouraging results. One important consideration here is the range of validity for these models. As pointed out by Seltz et al. [12], the networks are expected to have superior performance relative to the existing models for the conditions close to the data used for training.

Producing accurate training data is an important part of ML. In this regard, DNS as the most accurate technique for solving the governing equations can play a key role. However, one limitation for DNS is its high computational cost, in particular when the conditions are close to those in practical applications. The aim of this paper is therefore twofold. First, we present a computationally efficient solver for performing DNS of combusting flows. This combustion solver uses cylindrical coordinates and is therefore more efficient for round jet flames compared with existing DNS solvers that use Cartesian coordinates. Second, we will produce algebraic combustion models for LES using the GEP algorithm using the DNS data produced by our solver covering a large range of conditions on the premixed combustion regime diagram.

\section{Numerical methods \\ 1.1 DNS data}

The well-known high fidelity simulation solver HiPSTAR was used in this study [19]. HiPSTAR is capable of performing DNS and LES of compressible turbulent flows and has been used in numerous studies of non-reacting flows [20-22]. The code is parallelized for both central processing units (CPUs) and graphics processing units (GPUs). It is a multi-block, structured curvilinear solver and can solve the governing equations in a cylindrical coordinate system, enabling efficient distribution of grid points. The streamwise and radial directions are discretized using a fourth-order finite different scheme and Fourier decomposition is employed for discretization in the azimuthal direction. The coordinate mapping is restricted to two-dimensions, resulting in a smaller number of metric terms used in the solver. An axis treatment based on parity conditions is imposed [23]. Symmetric skew-splitting is applied to the convective terms of the governing equations to improve numerical stability [24].

This solver was recently modified such that it is now capable of performing combustion simulations using simple chemistry [25]. Numerous test cases were used for validation and 
a recent study investigating the impact of the inflow boundary conditions on flame characteristics of a turbulent premixed flame was undertaken [25].

This paper presents two DNS cases of statistically stationary turbulent methane-air premixed jet flames with the jet Reynolds number $R e_{D}=10,000$ at two different Karlovitz numbers. A schematic of the computational domain is shown in Figure 1. To reduce the computational cost, the domain is decomposed into different subdomains with different grid resolutions. Subdomain 1 features a turbulent pipe flow with a length of $25 D$. The unburnt gas was issued from the pipe into subdomain 2 with a turbulent flame anchored at the pipe lip. The flame was anchored using the temperature and progress variable profiles of a laminar premixed flame, with the same flame parameters as the turbulent cases, specified across the width of the lip. The pipe flow was fully coupled to the flame and therefore, there was no assumption around the flow length and time scales at the flame base. A coflow of hot burnt gases at the velocity of $1 \%$ of the jet bulk velocity entered through subdomains 5 and 7. A digital-filter based method was used to produce turbulent fluctuations at the pipe inlet and characteristics inflow boundary conditions were used at the co-flow boundary. Non-reflecting outflow boundary conditions were used at the outflow in the radial and streamwise directions [26].

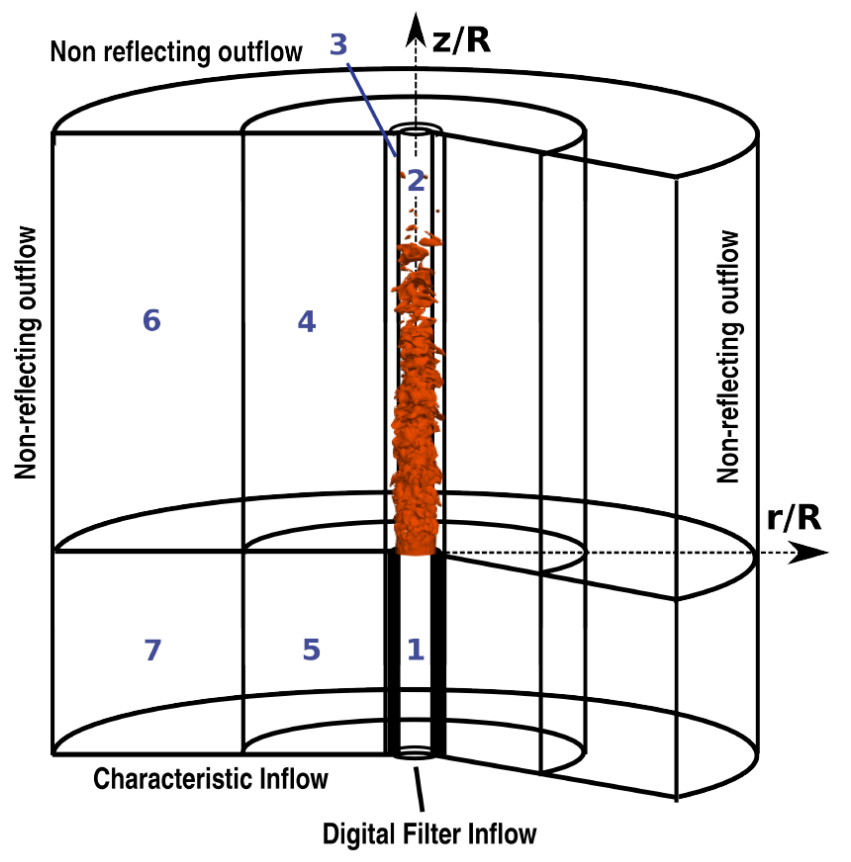

FIGURE 1. Schematic of the computational domain.

Table 1 presents the number of points in the streamwise and radial directions as well as the number of azimuthal Fourier modes. The grid is finest in the radial direction at the pipe lip so that the resolution at $r / R=1$ corresponds to at least 20 points across the thermal flame thickness. Polynomial stretching is applied with increasing radial and streamwise distance. In the azimuthal direction, the spacing in the physical coordinates corresponds to at least 6 collocation points per thermal flame thickness. More details of the computational domain can be found in [25].

\begin{tabular}{lccccc}
\hline Block & $l_{z}$ & $l_{r}$ & $N_{z}$ & $N_{r}$ & $k$ \\
\hline 1 & $50 / 50$ & $1 / 1$ & $560 / 560$ & $75 / 75$ & $192 / 128$ \\
2 & $60 / 100$ & $1 / 1$ & $2000 / 240$ & $75 / 75$ & $192 / 128$ \\
3 & $60 / 100$ & $0.1 / 0.1$ & $2000 / 2400$ & $25 / 25$ & $192 / 128$ \\
4 & $60 / 100$ & $7 / 17$ & $2000 / 2400$ & $200 / 290$ & $192 / 128$ \\
5 & $50 / 50$ & $7 / 17$ & $480 / 480$ & $200 / 290$ & $32 / 32$ \\
6 & $60 / 100$ & $37 / 27$ & $2000 / 2400$ & $300 / 240$ & $8 / 8$ \\
7 & $50 / 50$ & $37 / 27$ & $480 / 480$ & $300 / 240$ & $8 / 8$ \\
\hline
\end{tabular}

TABLE 1. Dimensions, grid points and Fourier modes for Flame-lowKa/Flame-high-Ka.

The simulation parameters of the two flames labeled as "Flame-low-Ka" and "Flame-high-Ka" are summarized in Table 2. Both flames have the same inlet velocity but different inlet temperatures. As a result, the laminar flame speed is lower for the case with lower inlet temperature. This leads to a higher Karlovitz number due to a smaller $S_{L}$ and therefore a higher $u^{\prime} / S_{L}$.

Single-step chemistry, representing the combustion of stoichiometric methane/air mixture at atmospheric pressure is used. This approach has been frequently used in the literature to study flame-turbulence interaction (e.g. [27-30]), as it features a much lower computational cost compared with detailed chemistry and can capture the general flame behavior in response to turbulence.

This chemical model is constructed such that the laminar flame speed, thermal flame thickness and the adiabatic flame temperature match those obtained from the detailed chemistry simulation of an unstrained laminar premixed flame with GRI 3.0. The Arrhenius law as a function of the obtained flame parameters such as $\alpha$ and $\beta$ (see Table 2) is used to calculate the reaction rates. Further details on the governing equations are presented in Ref. [25]. 


\begin{tabular}{lcc}
\hline Parameter & Flame-low-Ka & Flame-high-Ka \\
\hline$R e_{D}$ & 10000 & 10000 \\
$T_{u}$ & $800 \mathrm{~K}$ & $300 \mathrm{~K}$ \\
$M$ & 0.35 & 0.35 \\
$\Phi$ & 1.0 & 1.0 \\
$S_{L} / U_{b}$ & 0.00829 & 0.003 \\
$\delta_{t h} / D$ & 0.037 & 0.246 \\
$\delta_{z} / D$ & 0.0167 & 0.0463 \\
$\alpha$ & 0.675 & 0.865 \\
$K a$ & 4 & 90 \\
\hline
\end{tabular}

TABLE 2. Flame parameters.

\subsection{Gene-Expression Programming (GEP)}

As discussed earlier, GEP fits into the supervised learning group of ML, which expresses functional forms as sequences of variables and mathematical operators akin to sequences of genes that make up an individual. At each generation (iteration), a population of individuals is evaluated based on their fitness to the training data, and the concept of survival of the fittest is used to select a portion of the population that survives into the next generation. The new generation is repopulated by the replication of surviving individuals, while genetic variations, such as the processes of mutation and genetic crossover are introduced to the children to produce a diverse population. Then, individuals are once again set to compete against each other in an iterative process. In principle, GEP is a probabilistic search algorithm, where the best solutions are found by retaining better variations over successive iterations.

The workflow of the GEP-based symbolic regression is illustrated in Figure 2. First, a set of inputs is defined. This includes the definition of the symbols to be used, e.g., $+,-, \exp , x, y$, and the reading of the training data associated with the symbols $x$ and $y$. The probabilities of the genetic operations are defined, as well as the number of generations, population size, tournament size, number of genes per individual, genotype length, and convergence criteria. An initial population of individuals is then generated. The fitness of every individual is calculated using the training data. The convergence is then checked to see if any fitness is below the defined tolerance.

If the convergence criteria are met, the best solution is taken to be the output of the process. If convergence is not met, a selection (survival of the fitness) is done through the tournament selection process to pick out surviving individuals that are carried to the new generation. Genetic operators are then applied to create the new population. With the new population generated, the process loops back to begin the computation of the fitness again.

It is worth noting that the GEP settings need to be carefully chosen to find a suitable model in a computationally efficient manner. Important considerations include an appropriate representation of the initial population, a careful choice of the fitness function, an appropriate choice of the algorithm parameters such as the population size and genetic operation rate, and the high diversity of the population to avoid premature convergence [31-33].

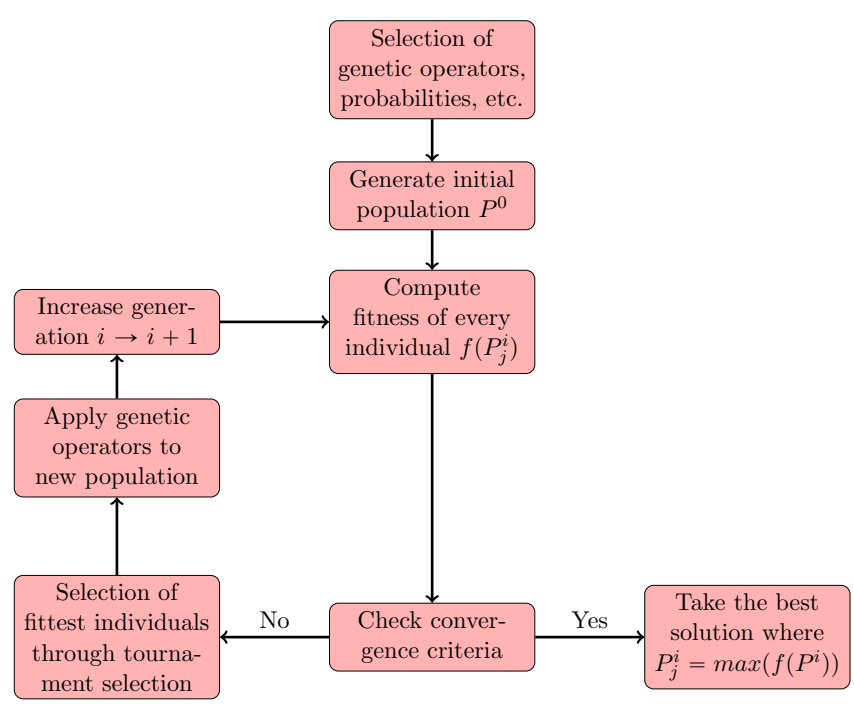

FIGURE 2. Work-flow of the GEP algorithm.

In the concept of flame surface density for combustion modeling, the unclosed reaction term is estimated using the following formulation,

$$
\overline{\omega_{\theta}}=\rho_{0}<S_{c}>_{s} \Sigma_{g e n},
$$

where $\dot{\omega}_{\theta}$ is the reaction rate for the reduced temperature, $\rho_{0}$ is the fresh gas temperature, $\left\langle S_{c}\right\rangle_{s}$ is the surface-averaged consumption speed and $\Sigma_{g e n}$ is the flame surface density. The variable $\Sigma_{\text {gen }}$ can then be modeled as,

$$
\Sigma_{g e n}=\Xi_{g e n}|\nabla \bar{c}|=f\left(\frac{\Delta}{\delta_{z}}, \frac{u_{\Delta}^{\prime}}{S_{L}}\right)|\nabla \bar{c}|
$$

where $\Xi_{g e n}$ is the flame wrinkling factor, $u_{\Delta}^{\prime}$ is the sub-grid scale velocity fluctuations and $\delta_{z}$ is the laminar Zel'dovich flame thickness. There are numerous algebraic models in the literature 


\begin{tabular}{ll}
\hline Name & Model formulation \\
\hline Charlette2 & $\Sigma_{\text {gen }}=\left(1+\min \left[\frac{\Delta}{\delta_{L}}, \Gamma_{\Delta}\left(\frac{u_{\Delta}^{\prime}}{S_{L}}\right)\right]\right)_{1}^{\beta}|\nabla \bar{c}| \quad$ where $\quad \Gamma_{\Delta}=$ \\
& {$\left[\left(\left(f_{u}^{-a_{1}}+f_{\Delta}^{-a_{1}}\right)^{-1 / a_{1}}\right)^{-b_{1}}+f_{R e}^{-b_{1}}\right]^{-1 / b_{1}}, \quad R e_{\Delta}=\frac{u_{\Delta}^{\prime} \Delta}{v}, \quad b_{1}=1.4$,} \\
& $\beta_{1}=0.5, C_{k}=1.5, a_{1}=0.60+0.2 \exp \left[-0.1 \frac{u_{\Delta}^{\prime}}{S_{L}}\right]-0.20 \exp \left[-0.01 \frac{\Delta}{\delta_{L}}\right]$, \\
& $f_{u}=4\left(\frac{27}{110} C_{k}\right)^{1 / 2}\left(\frac{18}{55} C_{k}\right)\left(\frac{u_{\Delta}^{\prime}}{S_{L}}\right)^{2}, \quad f_{\Delta}=\left\{\left(\frac{27}{110} C_{k} \pi^{4 / 3}\right)\left[\left(\frac{\Delta}{\delta_{L}}\right)^{4 / 3}-1\right]\right\}^{1 / 2}$, \\
\hline Fureby & $f_{R e}=\left[\frac{9}{55} \exp \left(-1.5 C_{k} \pi^{4 / 3} R e_{\Delta}^{-1}\right)\right]^{1 / 2} R e_{\Delta}^{1 / 2}$ \\
\hline Zimont & $\left.\left[1+0.51 \frac{u_{\Delta}^{\prime}}{S_{L}} \frac{3}{S_{L}} \frac{\Delta}{\delta_{L}}\right]^{\frac{1}{4}}\right]|\nabla \bar{c}|$ \\
\hline
\end{tabular}

TABLE 3. FSD models from the literature.

describing the wrinkling factor $\Xi_{g e n}$ as a function of $\Delta / \delta_{z}$ and $u_{\Delta}^{\prime} / S_{L}$ [34]. Some of these models, described in Table 2 will be used as a basis for testing the performance of the GEP approach. The objective of this study is to find an algebraic form for the function $f$ in equation 2. The fitness of the individuals were evaluated by minimizing the error between the conditional average of the training data and modeled results on the filtered progress variable:

$$
f i t=\dot{\Sigma} \int \frac{\left(\left\langle\Sigma_{\text {gen }} \mid \bar{c}, \Delta\right\rangle-\left\langle\Sigma_{\text {gen }}^{D N S} \mid \bar{c}, \Delta\right\rangle\right)^{2}}{\left\langle\Sigma_{\text {gen }}^{D N S} \mid \bar{c}, \Delta\right\rangle^{2}} d \bar{c},
$$

where $\Delta$ is the filter size.

Restrictions were placed on the minimum length of an individual to prevent premature sub-optimal convergence to overly simple solutions, and on the maximum number of nested operators to avoid excessively complex solutions. We also considered a maximum of 5000 generations and a population size of 5000 individuals. The tournament size was limited to 5 and a maximum length of 8 variables/operators were used for the GEP models.

\section{Results}

\subsection{DNS of turbulent premixed flames}

Figure 3 shows a visualization of the two flames by isosurfaces of the progress variable $c=0.87$. This value of $c$ corresponds to the point of the maximum heat release rate of a freely propagating premixed flame under the same conditions as the turbulent cases. As expected, Flame-high-Ka is much longer due to a smaller laminar flame speed. Furthermore, for this case, the presence of large scale structures at downstream locations can be inferred from the visualization of the flame surface.

The conditional means of the progress variable gradient across the flame brush for both flames are also shown in Figure 3 , along with the gradient for a freely propagating laminar flame under the same conditions as the turbulent cases for comparison. The higher gradients in Flame-low-Ka indicate a thinner flame brush compared to Flame-high-Ka. Close to the pipe exit at $z / L_{f}=0.04$, both flames show higher gradients in comparison to the laminar flame profiles due to the high tangential strain rate induced by the mean shear. At downstream locations, the gradients for Flame-low-Ka are the same as those in a laminar premixed flame, while Flame-high-Ka shows thickening of the flame brush due to distortion of the preheat zone by turbulent eddies.

The flame structures at three different streamwise locations, visualized by $\nabla c$ are shown in Figure 4. Highly curved flame surfaces experiencing small scale wrinkling are observed for Flamelow-Ka while larger scale wrinkling is present for Flame-highKa. This is consistent with the results presented in Figure 3, showing the presence of large structures at downstream locations for Flame-high-Ka.

Figure 5 shows the time and azimuthally averaged flame surface density of both flames. The jet half-width is also shown with a blue line. Flame-high-Ka features smaller gradients of the progress variable, consistent with the results shown previously. Furthermore, a stronger influence of the jet turbulence for this case leads to a thicker flame brush, even close to the inflow boundary. This is consistent with the results presented in Figure 4 , indicating that large structures evolved in the shear layer can still interact with the flame for Flame-high-Ka. Collectively, the results presented in Figures 3 to 5 suggest that these two cases feature different types of flame-turbulence interactions and are 


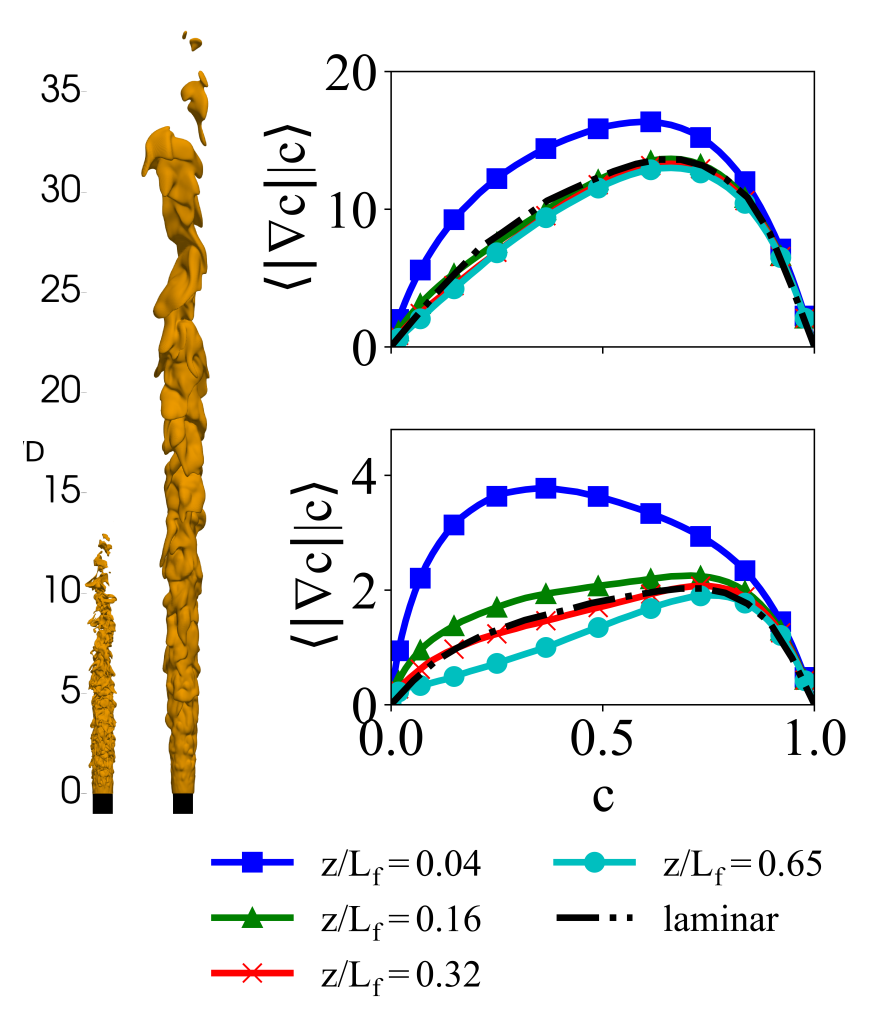

FIGURE 3. Visualization of Flame-low-Ka (left) and Flame-high-Ka (right) by iso-surface of $c=0.87$, and scalar gradient conditionally averaged on c for Flame-low-Ka (top) and Flame-high-Ka (bottom).

therefore suitable for training more general combustion models.

\subsection{Combustion modeling using GEP}

To train the GEP models for LES, the DNS results were first filtered using a Gaussian filter for different filter sizes $\Delta / \delta_{z}=1.7$, 2.2, 4.4, 8.8 for Flame-low-Ka and $\Delta / \delta_{z}=1.6,3.2,4.3,6.4$ for Flame-high-Ka. In this study, the training data consists of slices on the $r-\theta$ plane at several streamwise locations corresponding to $4 \%, 16 \%, 32 \%, 48 \%, 65 \%, 80 \%$, and $96 \%$ of their respective mean flame length $L_{f} / D$. A sampling of 100 time instants were used for training. Since the expected value of $\Sigma_{\text {gen }}$ for the unburnt and burnt mixtures was zero $(|\nabla \bar{c}|=0)$, only the data within the range of $0.01<\bar{c}<0.99$ was used for training. The variables $\Delta / \delta$ and the subgrid-scale velocity fluctuations $u_{\Delta}^{\prime}$ were input to the model for the wrinkling factor. The variable $u_{\Delta}^{\prime}$ was calculated from the sub-grid turbulent kinetic energy as,

$$
u_{\Delta}^{\prime}=\sqrt{\left(\widetilde{u_{i} u_{i}}-\widetilde{u_{i}} \widetilde{u_{i}}\right) / 3}
$$

In total, the number of data points in the training dataset was approximately 66 million.
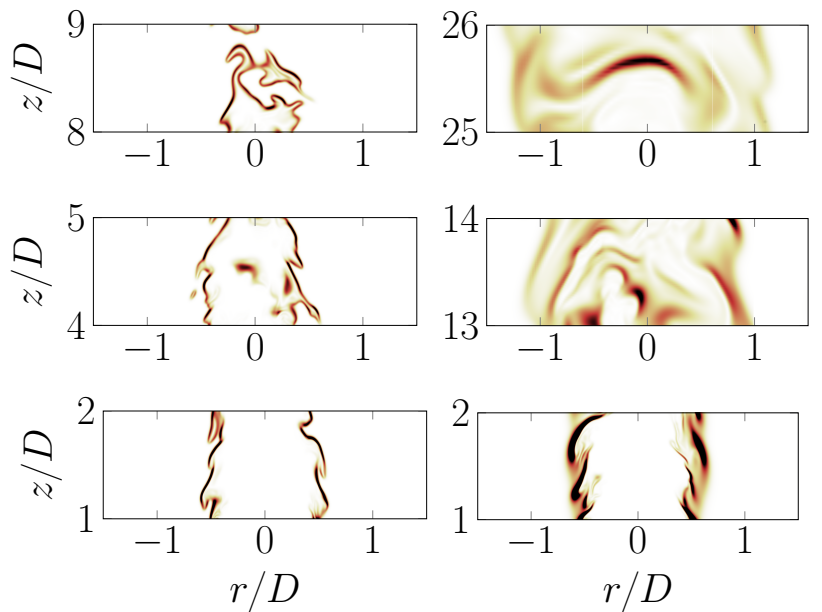

0.0

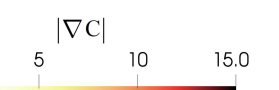

0.0

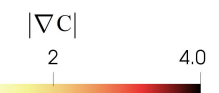

FIGURE 4. Slices of local scalar gradient for Flame-low-Ka (left) and Flame-high-Ka.
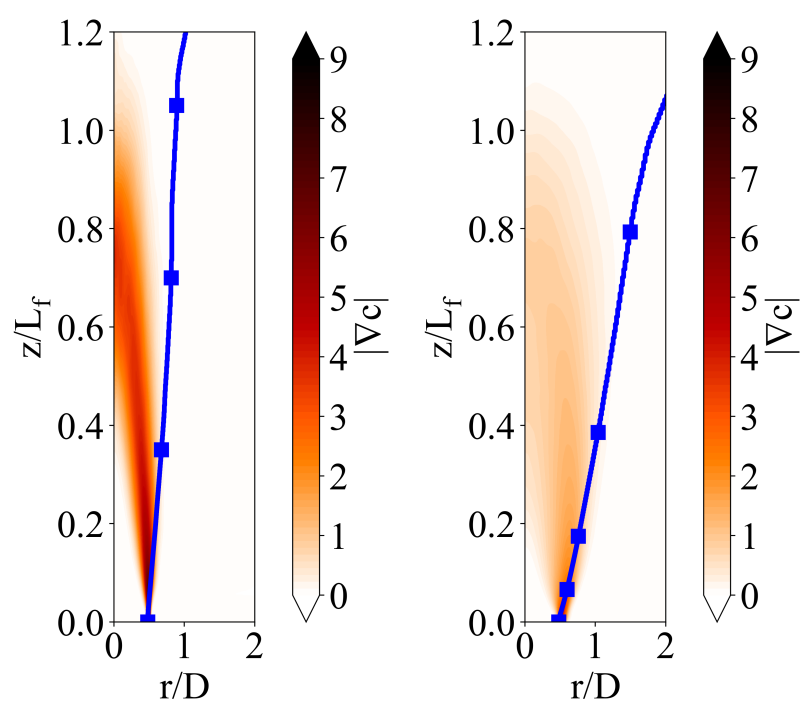

FIGURE 5. Time and azimuthally averaged surface density function for Flame-low-Ka (left) and Flame-high-Ka (right).

Figure 6 shows examples of the filtered DNS data for both flames. The thickening of the flame brush is observed as filter size increases. Furthermore, flame regions featuring high curvature values progressively smooth out for larger filter sizes.

Table 4 shows the three models generated using GEP denoted as GEPlow, trained using Flame-low-Ka only, GEPhigh, trained using Flame-high-Ka only and GEPall, trained using the data of both flames. GEPlow shows the lowest fitness value 


\begin{tabular}{lll}
\hline Name & Model formulation & fit \\
\hline GEPlow & $\Sigma_{\text {gen }}=0.636\left(u_{\Delta}^{\prime} / S_{L}+\left(2.189+\Delta / \delta_{z}\right)^{3}+\left(u_{\Delta}^{\prime} / S_{L}+3.934\right)^{1.5}\right)^{1 / 9}|\nabla \bar{c}|$ & 0.0031 \\
\hline GEPhigh & $\begin{array}{l}\Sigma_{\text {gen }}=1.413 \exp \left(1.742 u_{\Delta}^{\prime} / S_{L}^{2} / f_{1}^{2}\right)|\nabla \bar{c}| \quad \text { where } \quad f_{1}=2 u_{\Delta}^{\prime} / S_{L}-\Delta / \delta_{z}+ \\
\left(\left(\Delta / \delta_{z}-2.718\right)^{3}+2.478\right)^{0.5}+\left(u_{\Delta}^{\prime} / S_{L} /\left(\Delta / \delta_{z}-1\right)\right)^{2}\end{array}$ & 0.0224 \\
\hline GEPall & $\Sigma_{\text {gen }}=\exp \left(\frac{\left(\left(1-\Delta / \delta_{z}\right)^{2}\right.}{\Delta / \delta_{z}\left(14.301+\left(\Delta / \delta_{z}+u_{\Delta}^{\prime} / S_{L}^{3}\right)^{1 / 3}\right)}\right)|\nabla \bar{c}|$ & 0.0115 \\
\hline
\end{tabular}

TABLE 4. GEP generated models.
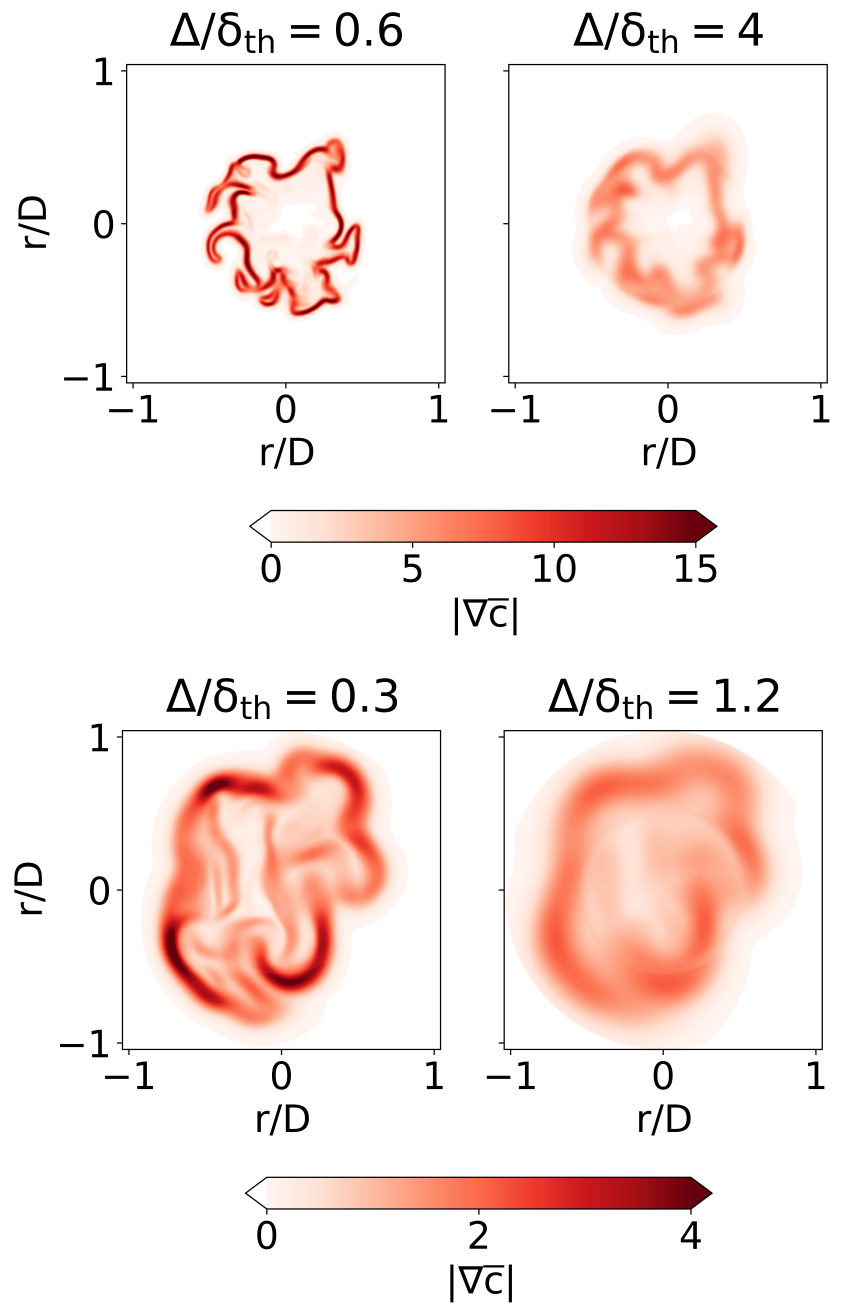

FIGURE 6. Filtered streamwise slice of Flame-low-Ka (top) and Flame-high-Ka (bottom) at $0.32 L_{f}$.

among all, indicating better fitness compared with the other two models.

Following the work of Chakraborty and Klein [34], the per- formance of the algebraic FSD models are assessed using three criteria: the variation of the volume-averaged FSD $\left\langle\Sigma_{g e n}\right\rangle$ with filter size, the correlation coefficient between the modeled FSD and FSD from the DNS data, and the conditionally averaged values of FSD $\left\langle\Sigma_{g e n} \mid \bar{c}\right\rangle$. The models are evaluated at streamwise locations and filter sizes different from the training data set: at locations corresponding to $10 \%, 40 \%$, and $70 \%$ of the respective mean flame length, and at filter sizes $\Delta / \delta_{z}=3,9$, and 12 . The GEP models are compared to models of similar form to Eq. 2, including Charlette2 [35], Fureby [36], and Zimont [37]. The models which use dynamic formulation, adjustable constants, or additional variables are excluded in the current paper as these features are not included in the current GEP formulations.

Figure 7 shows the variation of $\left\langle\Sigma_{g e n}\right\rangle$ with $\Delta / \delta_{z}$. The reference models generally overestimate $\left\langle\Sigma_{g e n}\right\rangle$ for both flames. For Flame-low-Ka, the Fureby model provides better estimates than the other reference models and gives reasonable estimates at $z / L=0.4$ and 0.85 . The GEP models are able to give better estimates of $\left\langle\Sigma_{g e n}\right\rangle$ compared to the reference models for both flames. Interestingly, while GEPlow shows a good performance for both flames, GEPhigh shows some discrepancy for small filter sizes for Flame-low-Ka.

Figure 8 shows the correlation coefficient between the DNS and the modeled FSD $\operatorname{corr}\left(\Sigma_{g e n}, \Sigma_{g e n}^{D N S}\right)$, where a unity value is desired. For large filter sizes, the GEP models show $\operatorname{corr}\left(\Sigma_{\text {gen }}, \Sigma_{\text {gen }}^{D N S}\right)$ closest to unity among all models. The Fureby model is the best out of the existing models for Flame-high-Ka. One should note that all models for $\Sigma_{g e n}$ are dependent on $|\nabla \bar{c}|$ through Eq. 2. Therefore, a high correlation between the predictions by the models and the DNS results does not necessarily demonstrate a good performance of the model for the wrinkling factor.

Figures 9 and 10 show the values of $\Sigma_{\text {gen }}$ conditionally averaged on $\bar{c}$ for both flames. The Charlette 2 and Zimont models are consistently overestimating $\left\langle\Sigma_{g e n} \mid \bar{c}\right\rangle$ for both flames. Consistent with the results shown in Figure 7 , the Fureby model provides the best estimates out of the reference models for Flame-low-Ka, at downstream locations. For Flame-high-Ka, all existing models overpredict $\left\langle\Sigma_{g e n} \mid \bar{c}\right\rangle$ for all filter sizes at all locations while the 


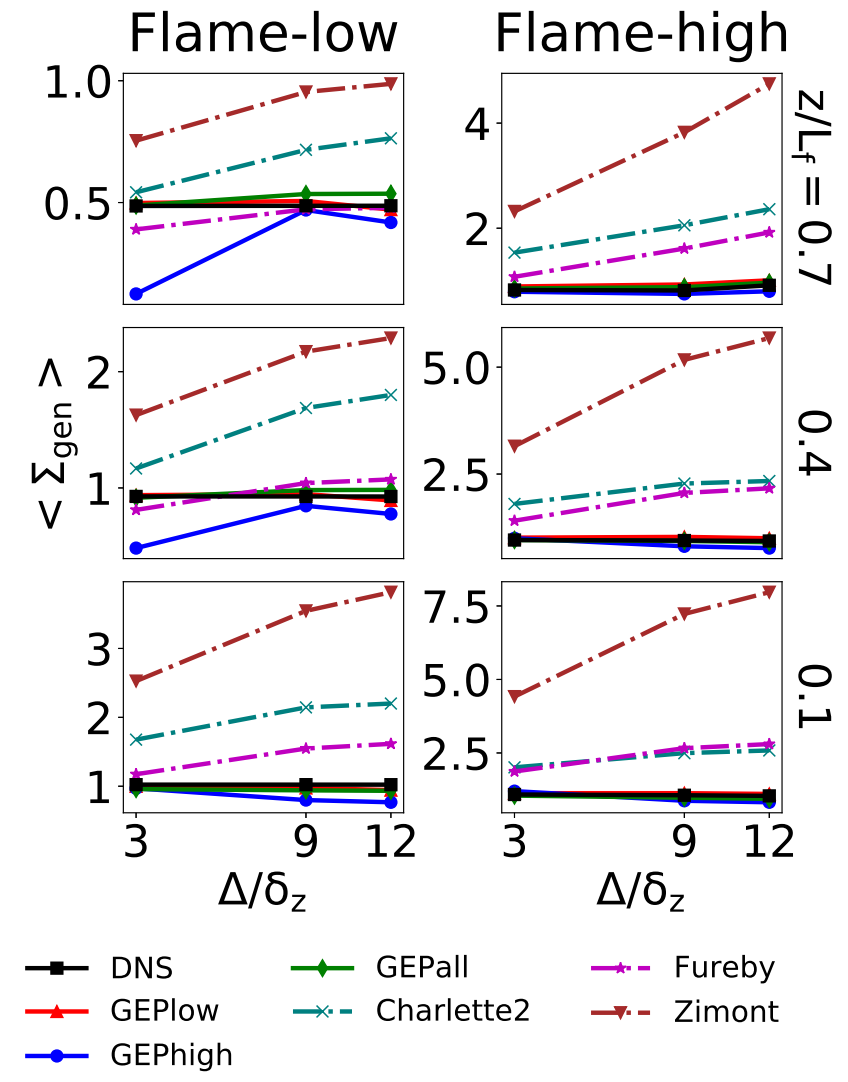

FIGURE 7. Variation of volume averaged FSD with filter size.

GEP models clearly show excellent performance. A comparison between the GEP models, once again shows that GEPlow has a large range of validity both in terms of the Karlovitz number as well as the filter size. One possible reason could be a larger range of $\left\langle\Sigma_{\text {gen }} \mid \bar{c}\right\rangle$ covered by Flame-low-Ka, making this case more suitable for training a universal model for FSD.

\subsection{Conclusions}

Gene-expression programming (GEP) was used to develop combustion models in the context of large-eddy simulation (LES). Direct numerical simulations (DNSs) of two turbulent premixed jet flames with different Karlovitz numbers were performed using a computationally efficient solver known as HiPSTAR. The filtered DNS data collected at different streamwise locations of the two flames were used to train algebraic flame surface density (FSD) models. Different subsets of the data were used to investigate how the choice of the training dataset impacts the performance of the GEP generated model. While the data of each flame was used to produce a dedicated GEP model, the third model was obtained using the data of both flames. The performance of the GEP models against some of the existing models in

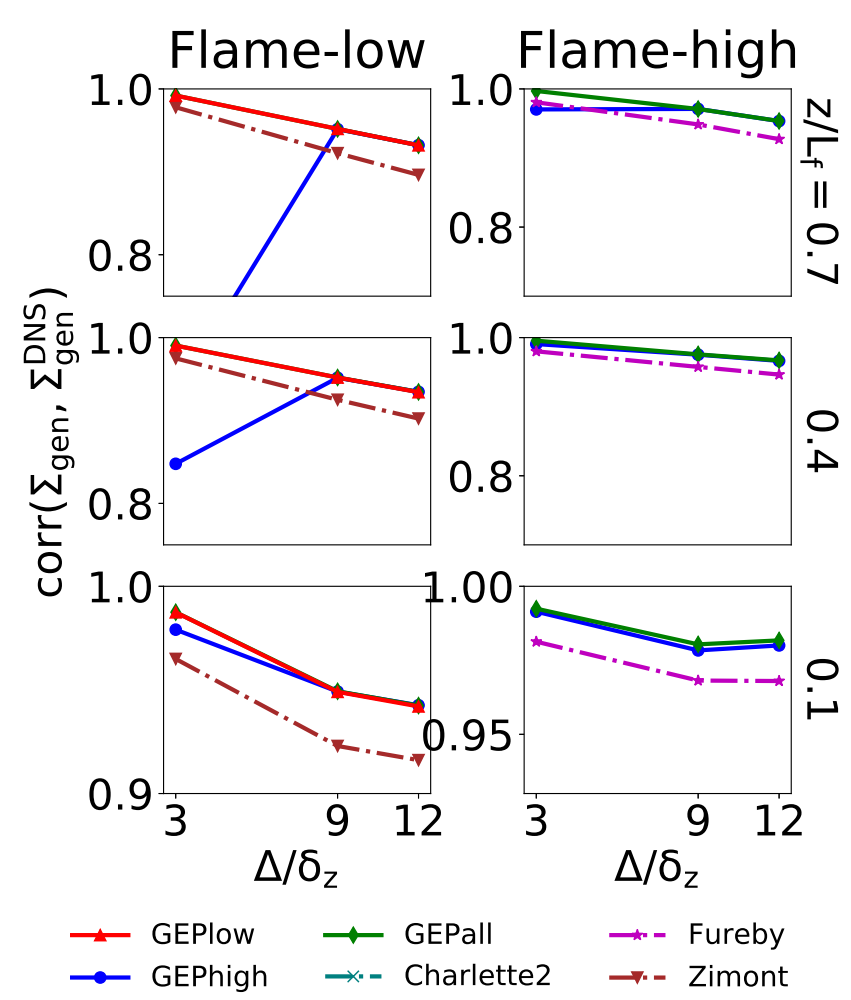

FIGURE 8. Correlation coefficient between DNS and modelled FSD in the range of $0.1<\bar{c}<0.9$.

the literature was then examined. The GEP models were found to have superior performance compared with the selected models from the literature. Furthermore, the GEP model trained by the low Karlovitz number flame only, was found more accurate for predicting the wrinkling factor for both low and high Karlovitz number flames. The results of this study show that GEP is a promising tool for developing algebraic FSD models for LES.

\section{ACKNOWLEDGMENT}

Mohsen Talei acknowledges the support of the Australian Research Council (ARC) [grant DE180100416]. This research was undertaken with the assistance of resources and services from the National Computational Infrastructure (NCI), which is supported by the Australian Government. This work was also supported by resources provided by the Pawsey Supercomputing Centre with funding from the Australian Government and the Government of Western Australia.

\section{REFERENCES}

[1] Mitchell, T., 1997. Machine learning. McGraw hill.

[2] Brunton, S. L., Noack, B. R., and Koumoutsakos, P., 2019. 


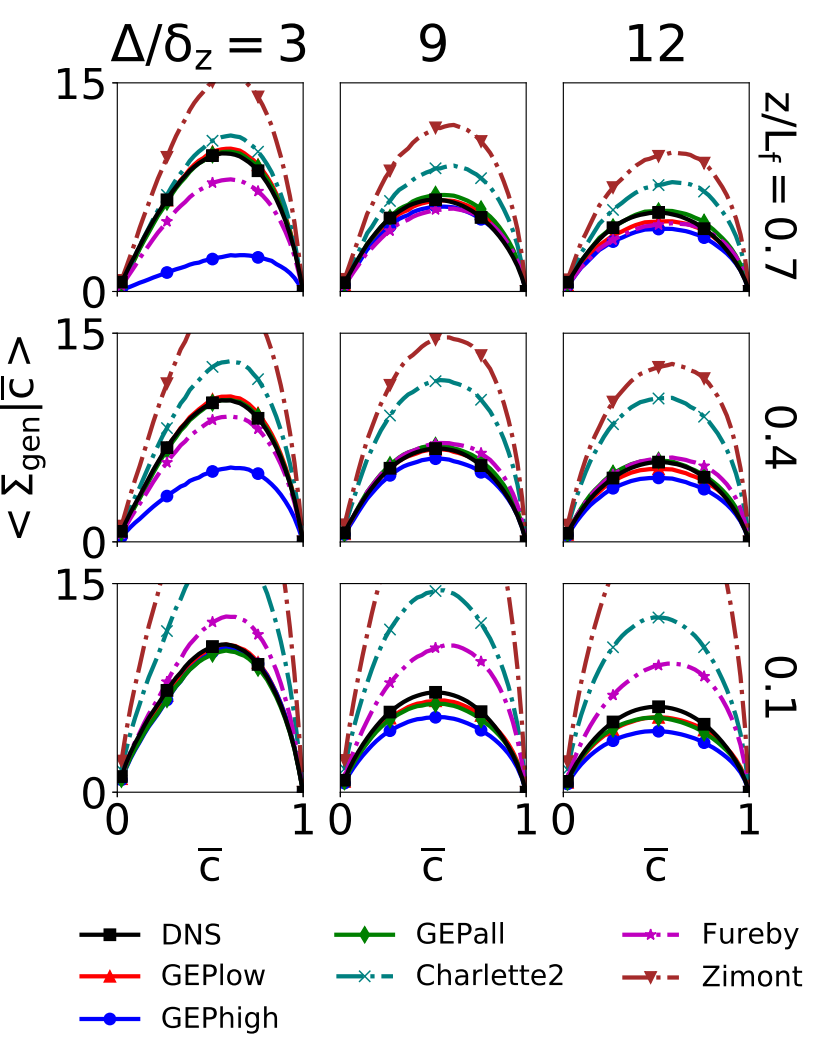

FIGURE 9. Conditional averaged FSD with $\bar{c}$ for Flame-low-Ka.

"Machine learning for fluid mechanics". Annual Review of Fluid Mechanics, 52.

[3] Tracey, B. D., Duraisamy, K., and Alonso, J. J., 2015. “A machine learning strategy to assist turbulence model development". In 53rd AIAA aerospace sciences meeting, p. 1287.

[4] Singh, A. P., Medida, S., and Duraisamy, K., 2017. "Machine-learning-augmented predictive modeling of turbulent separated flows over airfoils". AIAA Journal, pp. 2215-2227.

[5] Zhang, Z. J., and Duraisamy, K., 2015. "Machine learning methods for data-driven turbulence modeling". In 22nd AIAA Computational Fluid Dynamics Conference, p. 2460.

[6] Weatheritt, J., and Sandberg, R., 2016. "A novel evolutionary algorithm applied to algebraic modifications of the rans stress-strain relationship". Journal of Computational Physics, 325, pp. 22-37.

[7] Weatheritt, J., Pichler, R., Sandberg, R. D., Laskowski, G., and Michelassi, V., 2017. "Machine learning for turbulence model development using a high-fidelity hpt cascade simulation". In ASME Turbo Expo 2017: Turbomachinery Technical Conference and Exposition, American Society of

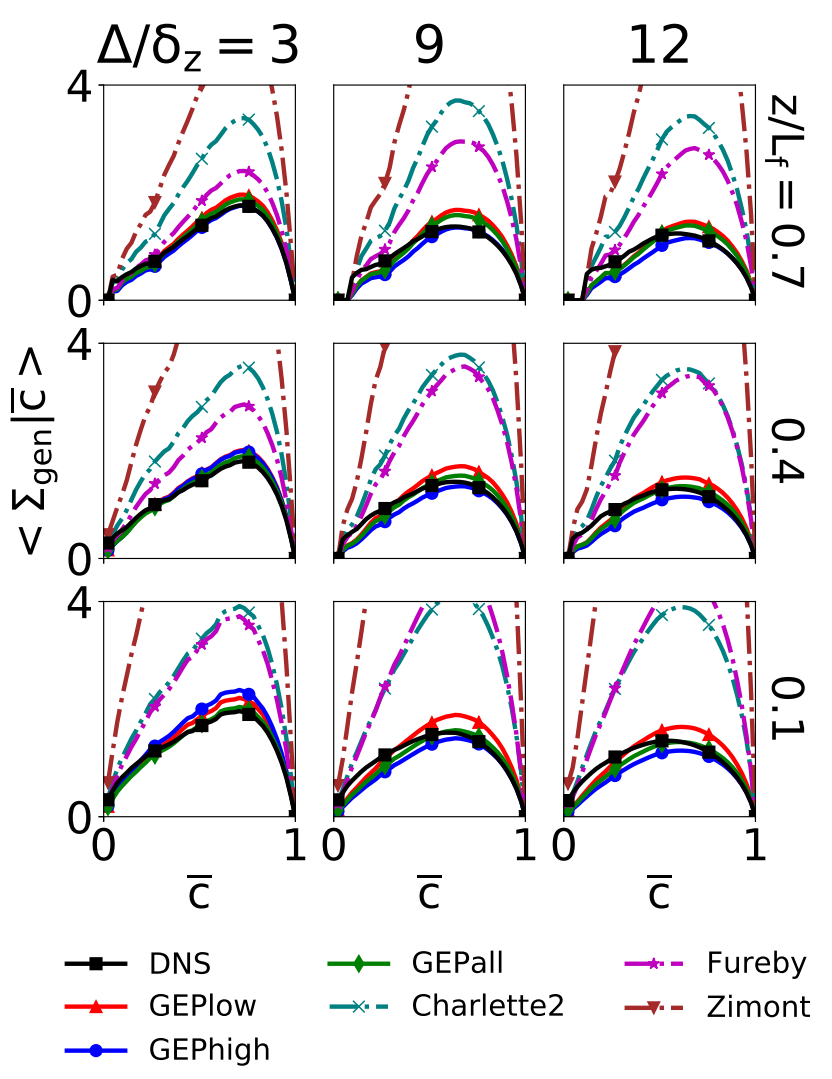

FIGURE 10. Conditional averaged FSD with $\bar{c}$ for Flame-high-Ka

Mechanical Engineers Digital Collection.

[8] Sandberg, R., Tan, R., Weatheritt, J., Ooi, A., Haghiri, A., Michelassi, V., and Laskowski, G., 2018. "Applying machine learnt explicit algebraic stress and scalar flux models to a fundamental trailing edge slot". Journal of Turbomachinery, 140(10), p. 101008.

[9] Schoepplein, M., Weatheritt, J., Sandberg, R., Talei, M., and Klein, M., 2018. "Application of an evolutionary algorithm to les modelling of turbulent transport in premixed flames". Journal of Computational Physics, 374, pp. 11661179.

[10] Lapeyre, C. J., Misdariis, A., Cazard, N., Veynante, D., and Poinsot, T., 2019. "Training convolutional neural networks to estimate turbulent sub-grid scale reaction rates". Combustion and Flame, 203, pp. 255-264.

[11] Hickey, J.-P., and Crowley, M. "Compact representation of a multi-dimensional combustion manifold using deep neural networks".

[12] Seltz, A., Domingo, P., Vervisch, L., and Nikolaou, Z. M., 2019. "Direct mapping from les resolved scales to filteredflame generated manifolds using convolutional neural networks". Combustion and Flame, 210, pp. 71-82. 
[13] Nunno, A. C., Perry, B. A., Macart, J. F., and Mueller, M. E., 2019. "Data-driven dimension reduction in turbulent combustion: Utility and limitations". In AIAA Scitech 2019 Forum, p. 2010.

[14] Ranade, R., and Echekki, T., 2019. "A framework for databased turbulent combustion closure: A posteriori validation". Combustion and Flame, 210, pp. 279-291.

[15] Ranade, R., and Echekki, T., 2019. "A framework for databased turbulent combustion closure: A priori validation". Combustion and Flame, 206, pp. 490-505.

[16] Shah, N., Zhao, P., DelVescovo, D., and Ge, H., 2019. Prediction of autoignition and flame properties for multicomponent fuels using machine learning techniques. Tech. rep., SAE Technical Paper.

[17] de Frahan, M. T. H., Yellapantula, S., King, R., Day, M. S., and Grout, R. W., 2019. "Deep learning for presumed probability density function models". arXiv preprint arXiv:1901.05557.

[18] Takbiri-Borujeni, A., and Ayoobi, M., 2019. "Application of physics-based machine learning in combustion modeling".

[19] Sandberg, R. D., 2012. "Numerical investigation of turbulent supersonic axisymmetric wakes". Journal of Fluid Mechanics, 702, pp. 488-520.

[20] Sandberg, R. D., Sandham, N. D., and Suponitsky, V., 2012. "DNS of compressible pipe flow exiting into a coflow". International Journal of Heat and Fluid Flow, 35, pp. 33-44.

[21] Sandberg, R., Michelassi, V., Pichler, R., Chen, L., and Johnstone, R., 2015. "Compressible Direct Numerical Simulation of Low-Pressure Turbines - Part I : Methodology". Journal of Turbomachinery, 137, p. 051011.

[22] Sandberg, R., and Tester, B., 2016. "Mach-number scaling of individual azimuthal modes of subsonic co-flowing jets". Journal of Fluid Mechanics, 793, pp. 209-228.

[23] Sandberg, R. D., 2011. "An axis treatment for flow equations in cylindrical coordinates based on parity conditions". Computers and Fluids, 49, pp. 166-172.

[24] Kennedy, C., and Gruber, A., 2008. "Reduced aliasing formulations of the convective terms within the Navier-Stokes equations for a compressible fluid". Journal of Computational Physics, 227, pp. 1676-1700.

[25] Ma, M., Talei, M., and Sandberg, R., In Press. "Direct numerical simulation of turbulent premixed jet flames: Influence of inflow boundary conditions". Combustion and Flame.

[26] Kim, J. W., and Lee, D. J., 2000. "Generalized characteristic boundary conditions for computational aeroacoustics". AIAA journal, 38(11), pp. 2040-2049.

[27] Haworth, D. C., and Poinsot, T., 1992. "Numerical simulations of lewis number effects in turbulent premixed flames". Journal of fluid mechanics, 244, pp. 405-436.

[28] Chakraborty, N., and Cant, R., 2009. "Direct numerical simulation analysis of the flame surface density transport equation in the context of large eddy simulation". Proceedings of the Combustion Institute, 32(1), pp. 1445-1453.

[29] Dunstan, T., Swaminathan, N., and Bray, K., 2012. "Influence of flame geometry on turbulent premixed flame propagation: a dns investigation". Journal of Fluid Mechanics, 709, pp. 191-222.

[30] Karami, S., Hawkes, E. R., Talei, M., and Chen, J. H., 2015. "Mechanisms of flame stabilisation at low lifted height in a turbulent lifted slot-jet flame". Journal of Fluid Mechanics, 777, pp. 633-689.

[31] Črepinšek, M., Liu, S.-H., and Mernik, M., 2013. "Exploration and exploitation in evolutionary algorithms: A survey". ACM computing surveys (CSUR), 45(3), pp. 1-33.

[32] Ferreira, C., 2006. Gene expression programming: mathematical modeling by an artificial intelligence, Vol. 21. Springer.

[33] Ursem, R. K., 2002. "Diversity-guided evolutionary algorithms". In International Conference on Parallel Problem Solving from Nature, Springer, pp. 462-471.

[34] Chakraborty, N., and Klein, M., 2008. "A priori direct numerical simulation assessment of algebraic flame surface density models for turbulent premixed flames in the context of large eddy simulation". Physics of Fluids, 20(8), p. 085108 .

[35] Charlette, F., Meneveau, C., and Veynante, D., 2002. “A power-law flame wrinkling model for les of premixed turbulent combustion part i: non-dynamic formulation and initial tests". Combustion and Flame, 131(1-2), pp. 159-180.

[36] Fureby, C., 2005. "A fractal flame-wrinkling large eddy simulation model for premixed turbulent combustion". Proceedings of the Combustion Institute, 30(1), pp. 593-601.

[37] Zimont, V., and Lipatnikov, A., 1995. "A numerical model of premixed turbulent combustion of gases". Chem. Phys. Rep, 14(7), pp. 993-1025. 


\section{University Library}

\section{- M M I N E R VA A gateway to Melbourne's research publications}

Minerva Access is the Institutional Repository of The University of Melbourne

Author/s:

Talei, M;Ma, D;Sandberg, R

Title:

Data-driven combustion modeling for a turbulent flame simulated with a computationally efficient solver

Date:

2020

\section{Citation:}

Talei, M., Ma, D. \& Sandberg, R. (2020). Data-driven combustion modeling for a turbulent flame simulated with a computationally efficient solver. Proceedings of ASME Turbo Expo 2020, 4A-2020, ASME: The American Society of Mechanical Engineers. https:// doi.org/10.1115/GT2020-14843.

Persistent Link:

http://hdl.handle.net/11343/258646 